\title{
3D view on Virgo and field dwarf elliptical galaxies: late-type origin and environmental transformations
}

\author{
Agnieszka Ryśs $\hat{s}^{1,2}$, Jesús Falcón-Barroso ${ }^{1,2}$ and Glenn van de Ven ${ }^{3}$ \\ ${ }^{1}$ Instituto de Astrofísica de Canarias, 38200 La Laguna, Tenerife, Spain \\ ${ }^{2}$ Departamento de Astrofísica, Universidad de La Laguna, 38205 La Laguna, Tenerife, Spain \\ ${ }^{3}$ Max Planck Institute for Astronomy, Königstuhl 17, 69117 Heidelberg, Germany \\ email: arys@iac.es
}

\begin{abstract}
In our contribution we show the effects of environmental evolution on cluster and field dwarf elliptical galaxies (dEs), presenting the first large-scale integral-field spectroscopic data for this galaxy class. Our sample con sists of 12 galaxies and no two of them are alike. We find that the level of rotation is not tied to flattening; we observe kinematic twists; we discover large-scale kinematically-decoupled components; we see varying gradient $\mathrm{s}$ in line-strength maps: from nearly flat to strongly peaked in the center. The great variety of morphological, kinematic, and stellar population parameters seen in our data supports the claim that dEs are defunct dwarf spiral/irregular galaxies and points to a formation scenario that allows for a stochastic shaping of galaxy properties. The combined influence of ram-pressure stripping and harassment fulfills these requirements, still, the exact impact of the two is not yet understood. We further investigate the properties of our sample by performing a detailed comprehensive analysis of its kinematic, dynamical, and stellar population parameters. The combined knowledge of the dynamical properties and star-formation histories, together with model predictions for different formation mechanisms, will be used to quant itatively determine the actual transformation paths for these galaxies.
\end{abstract}

Keywords. galaxies: dwarf - galaxies: evolution - galaxies: kinematics a nd dynamics

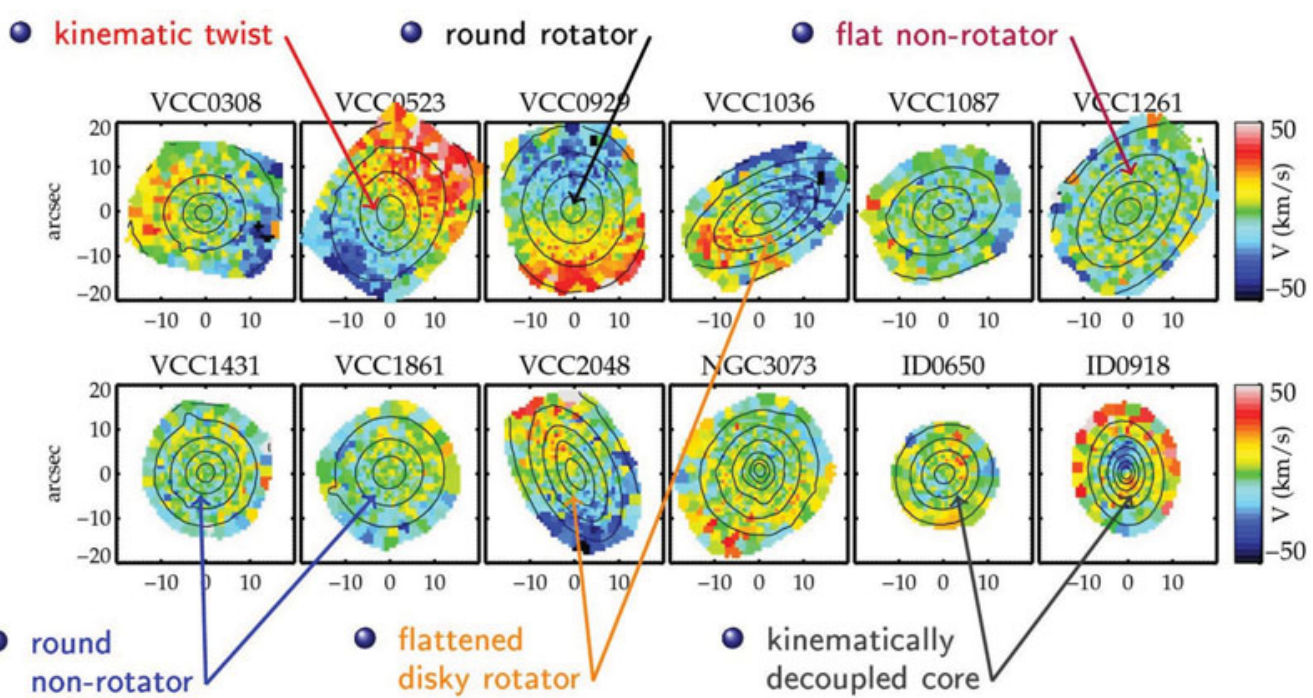

Figure 1. Stellar velocity maps showing the kinematic variety seen in our data. 